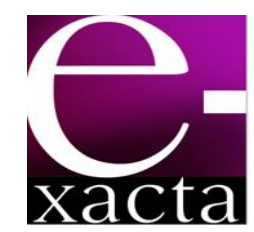

ISSN: 1984-3151

\section{ANÁLISE ESTRUTURAL DinÂMICA DE GRADE DE Proteção de Turbina de Usina Hidroelétrica}

Analysis Structural Dynamics of Grade Plant Turbine

Protection Hydroelectric

\author{
1 Engenheiro Mecânico. UNIFEI/MG, 2001. Mestre em \\ Engenharia Mecânica pela Universidade de Taubaté - \\ UNITAU, 2015. Professor Auxiliar de Resistência dos \\ Materiais da Universidade de Taubaté - UNITAU. Taubaté, \\ SP. lucasgiov@ig.com.br. \\ 2 Professor Doutor em Engenharia Mecânica. UNICAMP/SP, \\ 1990. Professor Assistente Doutor da Universidade de \\ Taubaté - UNITAU. Taubaté, SP. \\ carlosachaves@yahoo.com.br. \\ 3 Professor Doutor em Engenharia Aeronáutica e Mecânica. \\ ITA, 1997. Professor Assistente Doutor da Universidade de \\ Taubaté - UNITAU. Taubaté, SP. alvaro@unitau.br.
}

Lucas Giovanettii ; Carlos Alberto Chaves ${ }^{2}$; Álvaro Manoel de Souza Soares ${ }^{3}$

Recebido em: 09/05/2015 - Aprovado em: 01/03/2016 - Disponibilizado em: 31/05/2016

RESUMO: Grades são equipamentos de grande importância porque são responsáveis pela proteção das turbinas de usinas hidroelétricas contra impacto de corpos flutuantes. O objetivo do presente trabalho é analisar grades submetidas à ação de fluxo de água. Ou seja, analisar as respostas da estrutura e o comportamento do escoamento da água utilizando os cálculos de dinâmica de estruturas acopladas às técnicas de dinâmica de fluidos computacional (CFD), para um regime turbulento, mediante o uso do software comercial CFX versão 14. Tais análises são elaboradas mediante o processo de interação fluido-estrutura. Um modelo estrutural simplificado das barras verticais das grades é elaborado a partir de dados de projeto conhecidos. A partir desse modelo definese um volume de controle que representa o escoamento do fluido. Devido ao número de Reynolds calculado, utiliza-se o modelo de turbulência para obtenção dos resultados tais como tensões e deslocamentos nas barras verticais; e perfil de velocidades do escoamento.

PALAVRAS-CHAVE: Grade de proteção de turbina. Interação fluido-estrutura. Escoamento turbulento.

ABSTRACT: Tashracks are very important equipment because they are responsible for protecting turbines of hydroelectric plants against floating bodies. The objective of this study is to analyze trashracks submitted by action of water flow. In other words, to analyze the responses of the structure and the behavior of water flow using dynamic of structures calculations coupled with computational fluid dynamics techniques (CFD) for a turbulent regime, through the use of commercial software CFX version 14. This analysis is elaborated by the process of fluid structure interaction. A simplified structural model of vertical bars is defined from other similar projects. For this model is defined a volume of control that represents fluid flow. Due to the Reynolds number calculated, it is utilized a turbulence model in order to obtain the results. These results are: stresses and displacements of vertical bars; and profile of velocities of flow. The results are analyzed and discussed.

KEYWORDS: Trashracks. Fluid structure interaction. Turbulent flow. 


\section{INTRODUÇÃO}

Uma das mais importantes funções de grades de estruturas de tomadas d'água de usinas hidroelétricas é a proteção contra a entrada de corpos flutuantes que podem se chocar com as pás da turbina hidráulica. Devido à essa grande importância, a análise das estruturas deve ser estudada a fim de se evitar falhas. Devido ao tipo de carregamento, provocado pelo escoamento de fluido, a análise deve ser considerada com base na dinâmica de estruturas. Tal estudo de comportamento dinâmico pode ser validado utilizando o método de elementos finitos.

Muitas falhas ocorridas em grades de tomada d'água foram provocadas por problemas de vibração. Em Behring e Yeh (1980), uma série de falhas provocadas em grades são apresentadas. Todas elas provocadas por vibração. Em uma das grades analisadas, a causa das falhas foi atribuída preliminarmente a um problema de material com defeito. Após uma análise mais detalhada, percebeu-se que as barras danificadas se concentravam na região inferior, próximo a soleira das grades. Nessa região, altas velocidades localizadas do fluxo de água costumam existir. Após quatro anos de operação, notou-se que algumas barras verticais entre suportes horizontais haviam-se perdido nos painéis inferiores. Durante os trabalhos de reparo, um painel de grade em bom estado de conservação, localizado na parte superior foi substituído por um painel inferior. Depois de duas semanas, esse painel substituído perdeu duas barras verticais entre suportes e a terceira estava quebrada. Outras falhas são apresentadas por Behring e Yeh (1980), todas elas provocadas por vibração excessiva. A principal mais básica utilizada é que a região crítica é a região próxima a soleira, ou seja, onde existe maior velocidade do escoamento.

Em Nascimento, Silva e Giunta (2003), um modelo de grade de uma grande máquina de usina hidroelétrica considerando o problema de interação fluido-estrutura é apresentado. As características modais foram determinadas considerando a estrutura submersa na água e no ar. Além disso, o trabalho apresenta uma análise de verificação de riscos de danos provocados por sobrecarga devido à obstrução por plantas aquáticas e outros detritos acumulados entre as barras. Segundo os autores, as frequências naturais da estrutura quando a mesma está submersa na água são menores quando a estrutura está no ar. Esse fato pode ser comprovado através de fórmula apresentada pela norma ABNT NBR 11213 (2001). Tal norma apresenta uma expressão para determinação da primeira frequência natural das barras submersas em água. A partir dos resultados obtidos por esse cálculo da norma, é possível comparar com resultados da estrutura exposta ao ar livre.

Scheumann (2012) apresenta um estudo de caso detalhado de interação fluido-estrutura em grades. Nesse estudo, o objeto é a investigação de uma falha em das grades da tomada d'água da Usina Rock Island Dam. Nesse trabalho, a causa foi atribuída a um problema de corrosão. Mesmo assim, uma análise dinâmica foi elaborada para garantir que as tensões provocadas pela vibração não atingiram valores críticos.

Em Sadrnejad (2002), as cargas que provocam falhas em grades de natureza dinâmica são enfatizadas em vários estudos, ou seja, as características dinâmicas das estruturas das grades são analisadas. A solução clássica de massa concentrada aplicada à interação dinâmica fluido-estrutura é utilizada como um procedimento de solução aproximada do problema.

Em Nguyen, Naudascher e Fellow (1991), são apresentados vários estudos experimentais de escoamentos em barras de grades considerando fluxo paralelo e fluxo oblíquo. Nesse trabalho, é possível obter as respostas da amplitude de vibração em função da velocidade do escoamento, do ângulo de 
incidência e da geometria do corpo (que no caso é a seção retangular transversal das barras).

Kerenyi et al. (1997) descrevem os resultados experimentais de uma investigação de vibração provocada por escoamento de fluido em barras verticais. A identificação dos coeficientes de força dinâmica do fluido é determinada por meio de análise de regressão acompanhada por simulação numérica. Nesse trabalho é apresentado um modelo físico para o problema.

Hribernik e Hribernik (2013) apresentam uma investigação de diferentes tipos de projetos de grades e os impactos gerados nas perdas de carga dos escoamentos de fluido. Uma análise numérica em ANSYS CFX é elaborada para calcular a velocidade de aproximação do fluido. O objetivo é determinar um projeto otimizado da grade que minimiza a perda de carga, ou seja, maximiza a eficiência da máquina geradora.

NBR 11213 (2001) apresenta uma análise para verificação da vibração nas grades. Segundo essa norma, existe uma relação entre a frequência natural da grade e a frequência de excitação devido aos vórtices, ou seja, para um projeto seguro contra vibrações, a relação entre frequências deverá ser maior que 1,5 , com obstrução de $25 \%$ da área de passagem do fluido.

Em Kolkman e Jongeling (2007), uma excitação devido à pulsação periódica no fluxo gerado por bombas e turbinas (essa frequência é calculada através da velocidade de rotação dividido pelo número de pás) é apresentada. Essa excitação é uma causa de vibração em grades. Todos os tipos de danos devem ser observados. Porém, esses danos, na maioria dos casos, estão localizados nos painéis inferiores. Algumas barras se quebram por fadiga e outras simplesmente desaparecem. Alguns parafusos de fixação se rompem por tração e porcas desaparecem devido à vibração.
Em Ghamry e Katopodis (2012), são apresentados resultados de análises numéricas de fluxo turbulento escoando entre as barras verticais com diferentes geometrias e espaçamentos entre barras em condutos forçados. As simulações foram elaboradas utilizando duas classes de modelos de turbulência: o modelo de Navier-Stokes de Reynolds médio e o modelo de tensão de Reynolds. Alguns resultados experimentais foram utilizados para validar os modelos de turbulência. Análises quantitativas e qualitativas foram executadas comparando valores medidos e calculados dos modelos de turbulência.

Huang et al. (2013) apresentam uma análise modal da estrutura das grades e compara com dados experimentais.

Com o avanço das ferramentas computacionais, principalmente os aplicativos de cálculo estrutural em elementos finitos, atualmente é possível modelar estruturas otimizadas de forma a minimizar o peso. Entretanto, deve-se tomar um cuidado especial quando analisamos estruturas submetidas a cargas dinâmicas. Principalmente estruturas sujeitas ao escoamento de fluido.

O objetivo deste trabalho é analisar um modelo de grade de proteção de turbina hidroelétrica, submetida à ação de fluxo de água, analisando as respostas da estrutura e o comportamento do escoamento da água utilizando os cálculos de dinâmica de estruturas acopladas às técnicas de dinâmica de fluidos computacional (CFD), para um regime turbulento, mediante o uso do software comercial CFX versão 14.

\section{Modelo Físico e Matemático}

\subsection{DeSCRIÇÃo do MOdELO FísICO}

O problema aqui considerado é análise da estrutura sob ação do escoamento de um fluido, através da definição de um volume de controle. Esse volume foi definido de acordo com o tipo de utilização do 
equipamento. A Figura 1 apresenta uma vista em corte de uma usina hidrelétrica com os principais equipamentos.

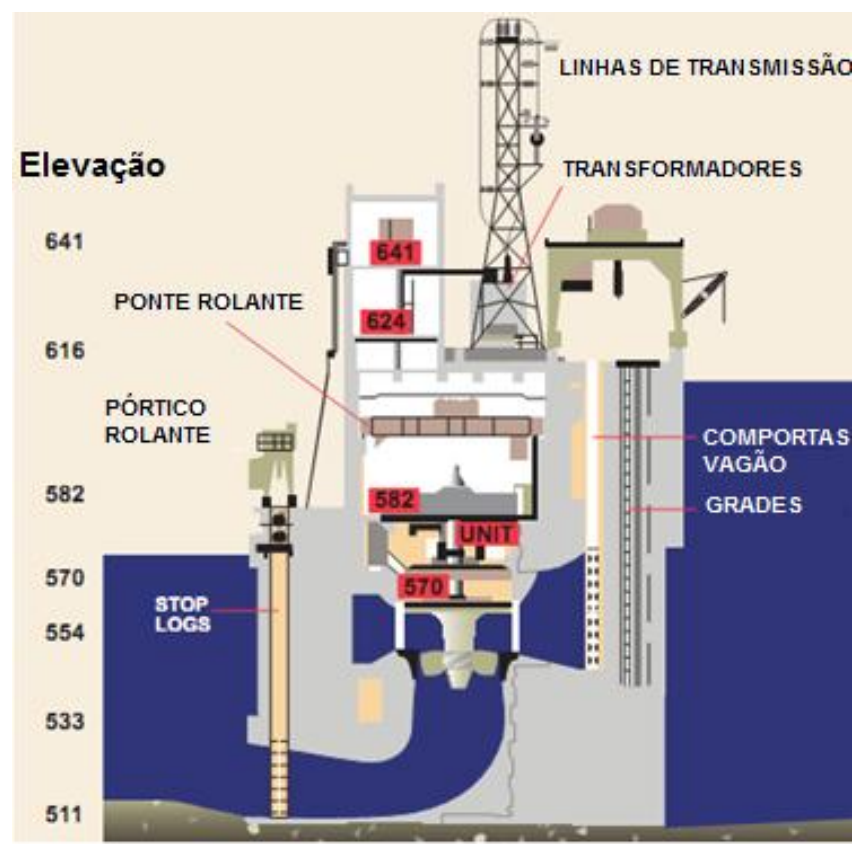

Figura 1 - Corte da Usina Hidrelétrica Fonte - Sheuman (2012)

A partir da análise da Figura 1, o próximo passo é analisar o equipamento hidromecânico cuja função é proteger a turbina do impacto de corpos flutuantes. $\mathrm{Ou}$ seja, definir uma geometria para analisar o problema. A Figura 2 apresenta uma geometria de grade que foi utilizada para definir o modelo do presente estudo.

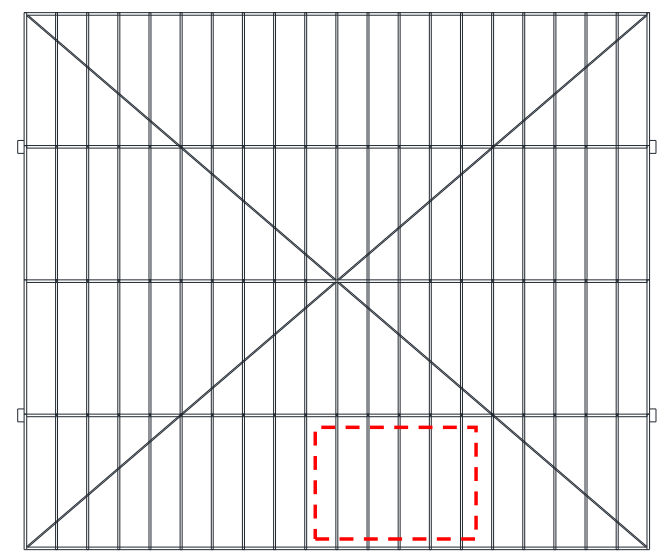

Figura 2 - Esquema da entrada do conduto com os painéis de grade Fonte - Próprio autor
A partir da Figura 2, definiu-se que o objeto da análise é a região mais próxima da soleira. Ou seja, a região com maior probabilidade de falha devido à vibração. De acordo com o presente estudo, as estruturas com maior índice de falhas por vibração são as barras verticais. Diante desse fato, é proposto um modelo conforme apresentado na Figura 3. Essa Figura apresenta a geometria das 5 barras verticais das grades com as seguintes dimensões: $650 \times 50 \times 9,5 \mathrm{~mm}$. A distância entre barras considerada é de $150 \mathrm{~mm}$. As dimensões do volume de controle são $6039 \times 900 \times 650$ $\mathrm{mm}$.

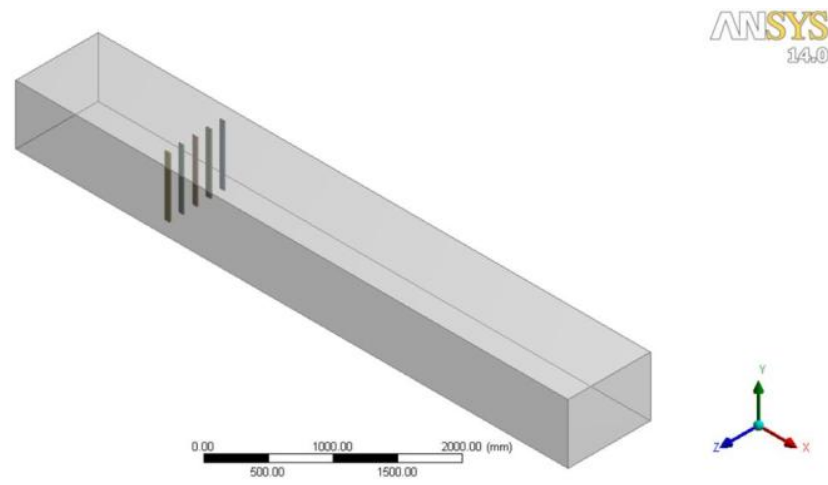

Figura 3 - Modelo do volume de controle e estrutura das barras verticais Fonte - Próprio autor

As dimensões do volume de controle foram definidas da seguinte forma. A distância a montante da grade foi considerada como $1510 \mathrm{~mm}$, que representa duas vezes o diâmetro hidráulico. Esse diâmetro hidráulico é definido pela Eq. 1.

$$
D_{\text {hidráulico }}=\frac{4 . \text { Área }}{\text { Perímetro }}
$$

A distância a jusante da grade foi considerada como 4529 mm, que representa seis vezes o diâmetro hidráulico. Esses dados foram definidos de forma a obter melhores resultados para o escoamento do fluido. 


\subsection{Descrição do Modelo Matemático (PARTe Estrutural)}

O modelo matemático que se aplica para este trabalho é de uma viga biengastada submetida à um carregamento distribuído. A Figura 4 apresenta o modelo estrutural simplificado utilizado no problema.

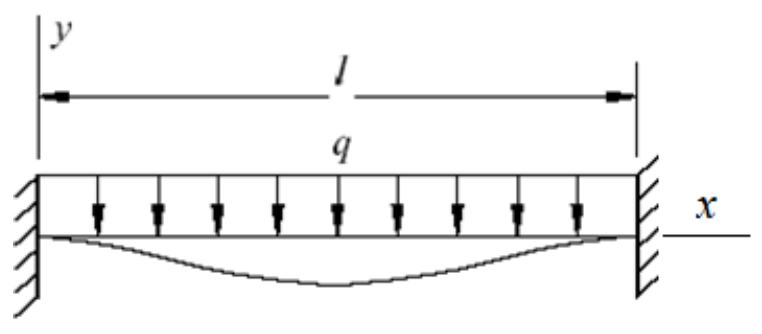

Figura 4 - Modelo estrutural das barras verticais Fonte - Próprio autor

Como a análise é de dinâmica de estruturas, a pressão dinâmica exercida pelo fluido representa a carga distribuída q que varia com o tempo. Segundo Timoshenko (1937), a equação diferencial da curva da deflexão estática de uma viga prismática é dada pela Eq. 2

$$
\text { E.I. } \frac{d^{4} y}{d x^{4}}=q
$$

onde $q$ representa a intensidade de carga distribuída e El é a rigidez a flexão da viga. Para adaptar essa equação para o caso de vibração lateral da viga, utiliza-se o princípio de D'Alembert e substitui-se a intensidade $q$ da carga lateral pela força de inércia por unidade de comprimento da viga, resultando na Eq. 3.

$$
E I \cdot \frac{\partial^{4} y}{\partial x^{4}}=-\frac{w}{g} \cdot \frac{\partial^{2} y}{\partial t^{2}}
$$

onde $g$ representa a aceleração da gravidade, $w / g$ representa a massa por unidade de comprimento da viga, $y$ representa a deflexão, $t$ representa a variável tempo, $x$ representa o eixo longitudinal da viga e El é a rigidez a flexão da viga.

\subsection{Descrição do Modelo Matemático (PARTe Hidráulica)}

O modelo matemático que governa o comportamento fluidodinâmico do escoamento de água no interior do volume de controle em estudo está constituído pela equação de continuidade, pela equação da quantidade de movimento, aplicadas a um escoamento laminar, isotérmico, tridimensional, incompressível, de um fluido viscoso, no caso água, com propriedades físicas constantes (BIRD; STEWART; LIGHTFOOT, 2004).

O sistema de equações solucionadas pela dinâmica de fluidos computacional são as equações de NavierStokes em sua forma conservativa e laminar, em estado estacionário (VERSTEEG; MALALASEKERA, 1995).

Para a formulação matemática do problema, as equações governantes correspondentes foram descritas em coordenadas cartesianas tridimensionais e consideraram-se as seguintes hipóteses: fluido newtoniano, escoamento turbulento, incompressível e em regime permanente com propriedades constantes. Adicionalmente, utilizou-se a hipótese de Boussinesq para descrever o termo de empuxo.

As equações da continuidade e da quantidade de movimento relacionadas ao escoamento estudado, são descritas, respectivamente, pelas Eq. 4 a Eq. 8.

Conservação da massa: A equação da conservação da massa (Eq. 4) assenta no princípio da continuidade e pode ser escrita da seguinte forma (BIRD, STEWART E LIGHTFOOT, 2004).

$$
\frac{\partial(\rho \cdot u)}{\partial x}+\frac{\partial(\rho \cdot v)}{\partial y}+\frac{\partial(\rho \cdot w)}{\partial z}=0
$$

onde $\rho$ é a massa específica do fluido de trabalho, u a componente do vetor velocidade na direção do eixo $\mathrm{x}$, $v$ a componente do vetor velocidade na direção do eixo y e w a componente do vetor velocidade na direção do eixo $z$. 


\section{Conservação da quantidade de movimento: As} equações de conservação da quantidade de movimento (Eq. 5), também conhecidas como equações de Navier-Stokes, seguem o princípio da segunda Lei de Newton: A variação de momentum em todas as direções é igual à soma das forças que atuam nessas mesmas direções (BIRD, STEWART E LIGHTFOOT, 2004).

$\frac{\partial(\rho \cdot u \cdot u)}{\partial x}+\frac{\partial(\rho \cdot v \cdot u)}{\partial y}+\frac{\partial(\rho \cdot w \cdot u)}{\partial z}=-\frac{\partial P}{\partial x}+\mu \cdot\left[\frac{\partial^{2} u}{\partial x^{2}}+\frac{\partial^{2} u}{\partial y^{2}}+\frac{\partial^{2} u}{\partial z^{2}}\right]+\rho \cdot g_{x}$

$$
\begin{aligned}
& \frac{\partial(\rho \cdot u \cdot v)}{\partial x}+\frac{\partial(\rho \cdot v \cdot v)}{\partial y}+\frac{\partial(\rho \cdot w \cdot v)}{\partial z}=-\frac{\partial P}{\partial y}+\mu \cdot\left[\frac{\partial^{2} v}{\partial x^{2}}+\frac{\partial^{2} v}{\partial y^{2}}+\frac{\partial^{2} v}{\partial z^{2}}\right]+\rho \cdot g_{y} \\
& \frac{\partial(\rho \cdot u \cdot w)}{\partial x}+\frac{\partial(\rho \cdot v \cdot w)}{\partial y}+\frac{\partial(\rho \cdot w \cdot w)}{\partial z}=-\frac{\partial P}{\partial z}+\mu \cdot\left[\frac{\partial^{2} w}{\partial x^{2}}+\frac{\partial^{2} w}{\partial y^{2}}+\frac{\partial^{2} w}{\partial z^{2}}\right]+\rho \cdot g_{z}
\end{aligned}
$$

onde $\rho$ é a massa específica do fluido de trabalho, u a componente do vetor velocidade na direção do eixo $\mathrm{x}$, $v$ a componente do vetor velocidade na direção do eixo y e w a componente do vetor velocidade na direção do eixo $z, \mu$ a viscosidade dinâmica, $g_{x}, g_{y}$ e $g_{z}$ as componentes do vetor aceleração da gravidade nas direções, respectivamente, $x, y$ e $z$ e $P$ a pressão.

O modelo Standard $k-\varepsilon$ tornou-se popular na solução de problemas de engenharia envolvendo escoamentos de fluidos com transferência de calor conjugada, por ser robusto, computacionalmente econômico e com precisão de resultados razoável em grande variedade de casos práticos. $O$ modelo Standard $k-\varepsilon$ é um modelo de duas equações semi-empírico, onde são introduzidas duas novas variáveis para a modelagem do escoamento, sendo elas, a energia cinética gerada pela turbulência $(k)$ e a sua taxa de dissipação $(\varepsilon)$. Esse modelo apenas é válido para escoamentos turbulentos completamente desenvolvidos e a influência da viscosidade molecular é desprezada.
A equação de transporte (Eq. 6) utilizada para modelar ké:

$\frac{\partial(\rho \cdot k \cdot u)}{\partial x}+\frac{\partial(\rho \cdot k \cdot v)}{\partial y}+\frac{\partial(\rho \cdot k \cdot w)}{\partial z}=\frac{\partial}{\partial x}\left[\left(\mu+\frac{\mu_{t}}{\sigma_{k}}\right) \cdot \frac{\partial k}{\partial x}\right]+\frac{\partial}{\partial y}\left[\left(\mu+\frac{\mu_{t}}{\sigma_{k}}\right) \cdot \frac{\partial x}{\partial y}\right]+\ldots$

$\ldots+\frac{\partial}{\partial z}\left[\left(\mu+\frac{\mu_{t}}{\sigma_{k}}\right) \cdot \frac{\partial k}{\partial z}\right]+G_{k}-\rho \cdot \varepsilon-Y_{M}+S_{k}$

A equação de transporte (Eq. 7) utilizada para modelar $\varepsilon$ é:

$\frac{\partial(\rho \cdot \varepsilon \cdot u)}{\partial x}+\frac{\partial(\rho \cdot \varepsilon \cdot v)}{\partial y}+\frac{\partial(\rho \cdot \varepsilon \cdot w)}{\partial z}=\frac{\partial}{\partial x}\left[\left(\mu+\frac{\mu_{t}}{\sigma_{\varepsilon}}\right) \cdot \frac{\partial \varepsilon}{\partial x}\right]+\frac{\partial}{\partial y}\left[\left(\mu+\frac{\mu_{t}}{\sigma_{\varepsilon}}\right) \cdot \frac{\partial \varepsilon}{\partial y}\right]+\ldots$
$\ldots+\frac{\partial}{\partial z}\left[\left(\mu+\frac{\mu_{t}}{\partial \varepsilon}\right) \cdot \frac{\partial \varepsilon}{\partial z}\right]+C_{1 \varepsilon} \cdot \frac{\varepsilon}{k} \cdot G_{k}-C_{2 \varepsilon} \rho \cdot \frac{\varepsilon^{2}}{k}+S_{\varepsilon}$

Nessas equações, $G_{k}$ representa a geração de energia cinética turbulenta devida aos gradientes da velocidade média. Por outro lado, $G_{M}$ diz respeito à energia cinética turbulenta gerada devido a efeitos de flutuabilidade. $Y_{M}$ representa a contribuição da dilatação flutuante em turbulência compressível.

$G_{1 \varepsilon}, G_{2 \varepsilon}$ e $G_{3 \varepsilon}$ são constantes que foram determinadas experimentalmente para ar e água em várias configurações de fluxo, enquanto $\sigma_{k}$ e $\sigma_{\varepsilon}$ são números de Prandtl turbulentos para $k$ e $\varepsilon$ respectivamente e assumem os valores apresentados na Tabela 1.

Tabela 1

Coeficientes do modelo Standard $k-\varepsilon$

\begin{tabular}{c|c}
\hline Coeficiente & Valor \\
\hline $\mathrm{C}_{1 \varepsilon}$ & 1,44 \\
$\mathrm{C}_{2 \varepsilon}$ & 1,92 \\
$\mathrm{C}_{\mathrm{u}}$ & 0,09 \\
$\sigma_{\mathrm{k}}$ & 1,0 \\
$\sigma_{\varepsilon}$ & 1,3 \\
\hline
\end{tabular}

Fonte - Bird, Stewart e Lightfoot (2004) 
Relativamente à viscosidade turbulenta, pode ser determinada pela Eq. 8 .

$\mu_{\mathrm{t}}=\rho \cdot \mathrm{C}_{\mathrm{u}} \frac{\mathrm{k}^{2}}{\varepsilon}$

O fluido newtoniano água pode ser considerado incompressível (Tabela 2). A temperatura de entrada do fluido água é $25^{\circ} \mathrm{C}$.

Tabela 2

Características do fluido água utilizado

\begin{tabular}{c|c}
\hline Variável & Valor \\
\hline $\begin{array}{c}\text { Densidade }\left(\mathrm{kg} \cdot \mathrm{m}^{-3}\right) \\
\text { Capacidade térmica específica } \\
\left(\mathrm{J} .(\mathrm{kg} \cdot \mathrm{K})^{-1}\right)\end{array}$ & 997 \\
Pressão de referência $(\mathrm{atm})$ & 1 \\
Temperatura de referência $\left({ }^{\circ} \mathrm{C}\right)$ & 25 \\
Viscosidade dinâmica $\left(\mathrm{kg} \cdot(\mathrm{m} . \mathrm{s})^{-1}\right)$ & $8,899 \times 10^{-4}$ \\
Condutividade térmica $\left(\mathrm{W} .(\mathrm{m} . \mathrm{K})^{-1}\right)$ & 0,6069 \\
\hline
\end{tabular}

Fonte - Próprio autor

\section{MÉTOdO}

\subsection{Ferramenta Computacional Utilizada e COMPUTADOR}

O software comercial CFX, em sua versão 14, é um programa para a predição de escoamento laminar e turbulento, e transferência de calor, massa e reações químicas, junto com modelos adicionais tais como escoamento multifásico, combustão e transporte de partículas. É baseado no método dos volumes finitos idealizado por Patankar (1980).

O programa CFX consiste de um número de módulos: geometria (Ansys Design Modeler), geração da malha (Ansys Meshing), setup do modelo (Ansys CFX-Pré), solução (Ansys CFX Solver) e Pós-Processamento ou gráfico (Ansys CFX-Pós) (ANSYS CFX, 2011).

\subsection{Malha Utilizada (Modelo Estrutural)}

A malha do modelo estrutural apresenta 13140 nós e 1625 elementos. O tamanho do elemento é definido como $10 \mathrm{~mm}$. A Figura 5 apresenta a malha de elementos finitos do modelo estrutural.
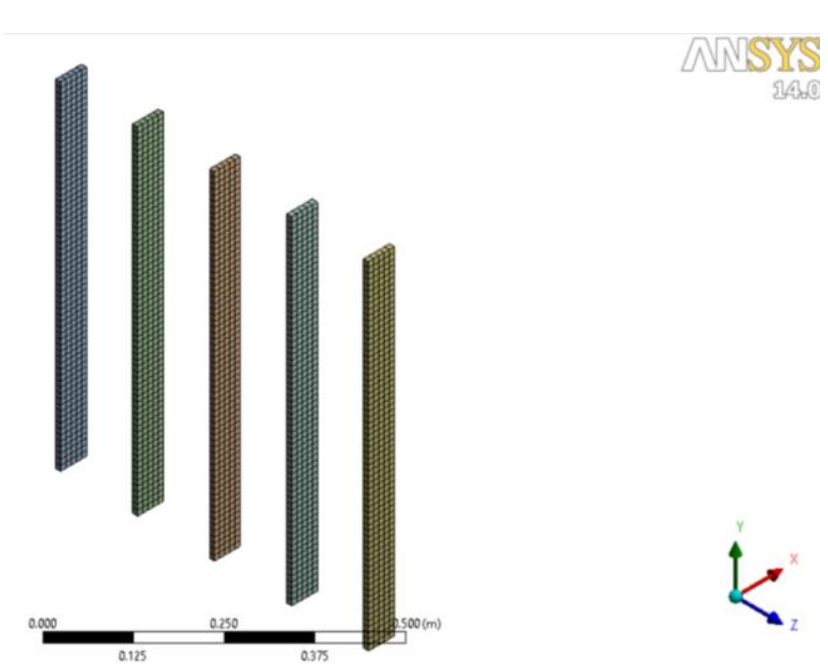

Figura 5 - Malha de elementos finitos das barras verticais

Fonte - Próprio autor

\subsection{Malha Utilizada (Modelo hidráUlico)}

Depois de definida a malha da parte estrutural, é possível definir a malha do domínio. É importante salientar que a escolha de uma boa malha é fundamental para a precisão dos resultados de dinâmica dos fluidos computacional. Sabendo que se trata do escoamento de um fluido é importante que o alinhamento da malha siga a direção do escoamento, o que é conseguido mais efetivamente com prismas ou hexaedros.

É altamente recomendável utilizar elementos hexaédricos próximos à parede, que neste caso é a região próxima às barras das grades. Não é interessante quando o fluxo passa por elementos triangulares próximos à parede. Isso se deve ao fato da Lei de Parede. O ajuste da malha é fundamental para a análise de CFD.

Tendo em conta as considerações anteriores, partiuse então para a produção da malha com recurso ao 
Meshing do pacote ANSYS WorkBench. Inicialmente foi criada uma malha com base em dados apresentados na Tabela 3.

Tabela 3

Características da malha gerada pelo Ansys

\begin{tabular}{c|c} 
& Meshing \\
\hline Variável & Condição \\
\hline $\begin{array}{c}\text { Preferência física } \\
\text { Método de geração } \\
\text { de malha }\end{array}$ & Método de varredura \\
\hline
\end{tabular}

Fonte - Próprio autor

A malha obtida com as definições referidas na Tabela 3 é mostrada nas Figuras 6 e 7. Observa-se que 0 software, definiu hexaedros para a geometria das células e distribuiu-as já na direção do escoamento. Essa malha apresentada possui 4.129 .028 nós e 3.965.280 elementos.

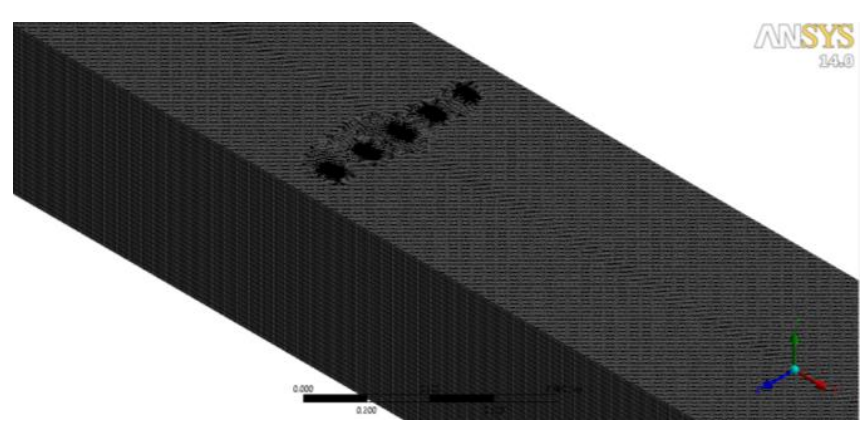

Figura 6 - Representação da malha (automática) do volume de controle usando o Ansys Meshing Fonte -: Próprio autor

Um detalhe da região da malha elaborada no Ansys Meshing do volume de controle é mostrado na Figura 7. É importante salientar que a malha sofreu um processo de refinamento na região de interesse. Ou seja, a malha foi refinada na região próxima a interface do fluido com a estrutura. $O$ processo de refinamento da malha se fez necessário para melhorar os resultados das análises. Os critérios de convergência da malha seguem os parâmetros padronizados do software ANSYS.

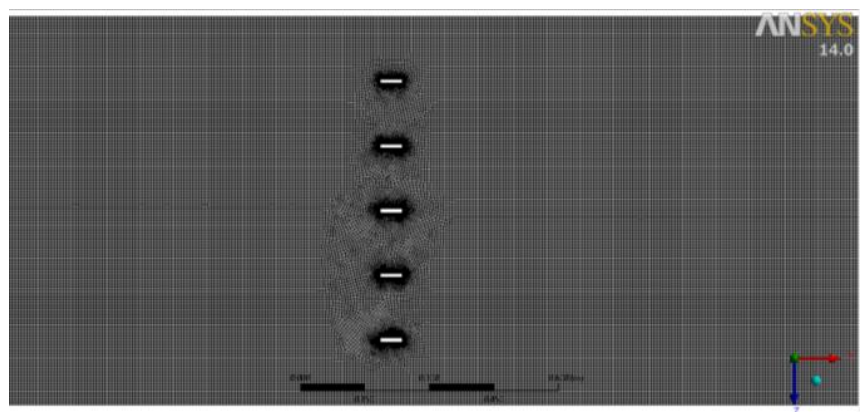

Figura 7 - Detalhe da malha do volume de controle usando o Ansys Meshing Fonte - Próprio autor

\subsection{Condições de Contorno (Modelo ESTRUTURAL)}

As condições de contorno do modelo estrutural são as seguintes: Engastamento perfeito nas extremidades das barras verticais. É aplicada à condição de interface fluido-estrutura na área molhada da grade. A interação entre o fluido e a estrutura na malha de interface provoca uma pressão que exerce uma força na estrutura. $E$ os movimentos estruturais produzem uma perturbação no escoamento. Esse mecanismo é cíclico e retroalimentado.

Além dessas condições, é aplicada a condição de carregamento através de uma pressão equivalente a 3,0 m.c.a. Essa pressão é definida conforme a norma ABNT NBR11213 (2001). A partir dessa pressão, determina-se a tabela de pressões que é inserida na análise. A Tabela 4 apresenta a variação de pressões em relação ao tempo, para uma análise de 5,0 segundos. A Figura 8 apresenta as condições de contorno aplicadas no modelo estrutural. 
Tabela 4

Tabela de carregamento

\begin{tabular}{c|c}
\hline Tempo (s) & Pressão (Pa) \\
\hline 0 & $4,6313 \times 10^{5}$ \\
0,499 & $4,6313 \times 10^{5}$ \\
0,5 & 0 \\
5 & 0 \\
\hline
\end{tabular}

Fonte - Bird, Stewart e Lightfoot (2004)

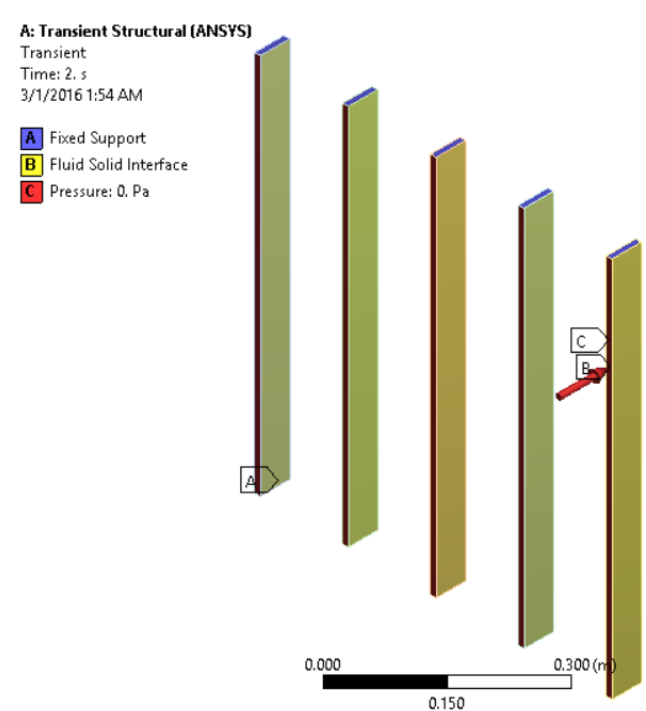

Figura 8 - Detalhe da malha do volume de controle usando o Ansys Wokbench

Fonte - Próprio autor

Devido à condição de carregamento, a análise estrutural é definida como transiente estrutural, ou seja, análise dinâmica.

\subsection{Condições de Fronteira (Modelo}

\section{HidRÁULICO)}

O software ANSYS CFX® versão 14 permite selecionar diversos tipos de condições de contorno como pressão, velocidade, vazão mássica, simetria, entre outras. Para as simulações realizadas neste trabalho, as principais condições de contorno utilizadas foram: condição de fronteira para escoamento na entrada, condição de fronteira para escoamento na saída e condição de simetria nas faces laterais do volume de controle (no plano perpendicular ao eixo $\mathrm{z}$ ).

Zona de entrada do fluido: Velocity flow inlet - neste tipo de condição de fronteira foi necessário definir a velocidade de entrada, a temperatura de entrada do fluido e para a situação de escoamento turbulento, também os valores de $k$ e $\varepsilon$. Os valores de $k$ e $\varepsilon$, poderiam ter sido calculados mas optou-se por gerar uma primeira simulação e ver para que valores estes tendiam, e numa segunda simulação atribuir esses valores no velocity flow inlet: $\operatorname{Red}=432.945$ (turbulento), $u=3,1 \mathrm{~m} / \mathrm{s}$;

\section{Zona correspondente as faces laterais do volume} de controle: Neste caso foi definida a condição de simetria no eixo $z$;

Zona de saída do fluido: Outflow - esta zona foi definida como saída de velocidade do fluido. Com a pressão de saída prescrita e igual a $(0 \mathrm{~Pa})$.

A Figura 9 apresenta as condições de fronteira aplicadas no modelo.

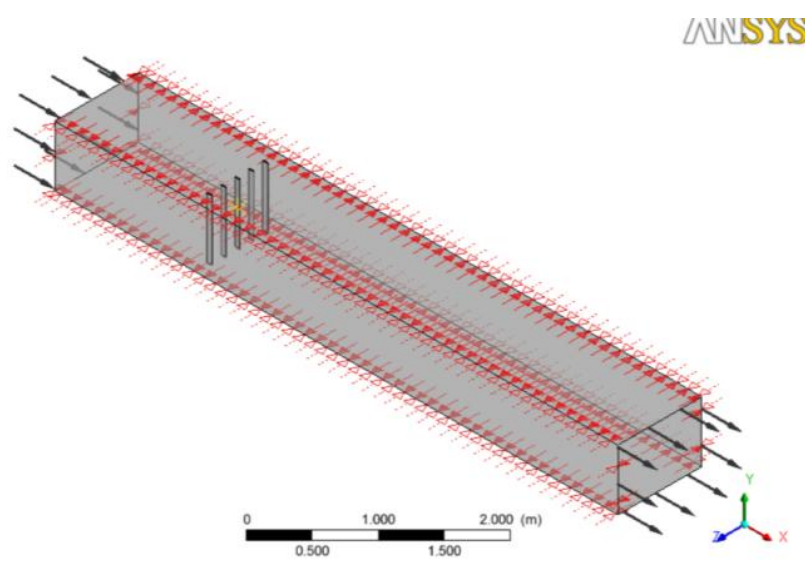

Figura 9 - Detalhe das condições de fronteira aplicadas ao modelo usando o Ansys CFX Fonte - Próprio autor 


\subsection{CondiçõEs ApLICADAS Às SimULAÇõES}

Na definição do modelo, se considera o fluxo de água como fluido. Em relação às simulações, a caracterização do modelo se definiu na etapa de PréProcessamento do software.

Considerou-se a opção de regime estacionário, devido ao fluido não variar suas propriedades com o tempo. No domínio estudado, especificou-se uma pressão de referência de $0 \mathrm{~Pa}$, na saída do escoamento.

O algoritmo de acoplamento, entre a pressão e velocidade, adotado para as simulações deste trabalho foi o SIMPLE (Semi-Implicit Method for Pressure-Linked Equations). O algoritmo SIMPLE é essencialmente um procedimento iterativo para prever e corrigir o cálculo do campo de pressão, cumprindo a conservação da massa (VERSTEEG; MALALASEKERA, 1995). As equações para as variáveis da solução são resolvidas sequencialmente e a solução é obtida iterativamente de forma a obter a convergência da solução.

O CFX resolve o processo iterativo da solução até que atinja determinados critérios de parada especificados. Esses critérios de parada são especificados para as equações da continuidade e energia e para as velocidades em $x, y, z$.

As condições impostas ao domínio do fluido requeridas para se definir a simulação são apresentadas na Tabela 5.
Tabela 5

Condições utilizadas para as simulações

\begin{tabular}{c|c}
\hline Parâmetro & Valor \\
\hline Tipo de simulação & Estacionário \\
Esquema advectivo & High resolution (default) \\
$\begin{array}{c}\text { Pressão de saída da } \\
\text { tubulação }\end{array}$ & $0 \mathrm{~Pa}$ \\
$\begin{array}{c}\text { Temperatura de } \\
\text { referência na } \\
\text { entrada } \\
\text { Critério de } \\
\text { convergência } \\
\text { Solução inicial }\end{array}$ & $25^{\circ} \mathrm{C}$ \\
$\begin{array}{c}\text { Timescale control } \\
\text { RMS (raiz do desvio } \\
\text { quadrático médio) }\end{array}$ & Automático \\
\hline
\end{tabular}

Fonte - Próprio autor

É necessário verificar se o escoamento é laminar ou turbulento. Foi então calculado o número de Reynolds através da Eq. 9.

$\operatorname{Re}=\frac{\rho \cdot \mathrm{u} \cdot \mathrm{D}}{\mu}$

Sendo $V=3,1 \mathrm{~m} / \mathrm{s}$, obtém $R e=432.945$, trata-se de um escoamento claramente turbulento, uma vez que em tubos a transição de escoamento laminar para turbulento dá-se para números de Re compreendidos entre 2.300 e 3.000 .

Considerando a pressão estática de 3 m.c.a., atuando em uma viga bi-engastada, a tensão exata é de 39,13 $\mathrm{MPa}$, nos apoios. A Equação 10 apresenta o cálculo do Momento Fletor na região dos apoios para o cálculo da tensão:

$M_{f}=\frac{q \cdot l^{2}}{12}$

Onde $q$ é a intensidade da carga distribuída e $l$ é o comprimento da barra. 


\section{Resultados e Discussão}

\subsection{Resultados da Análise Estrutural}

Os resultados das tensões de Von Mises obtidas estão apresentados na Figura 10. Os resultados apresentados são referentes à um intervalo de tempo igual a 0,4 segundos.
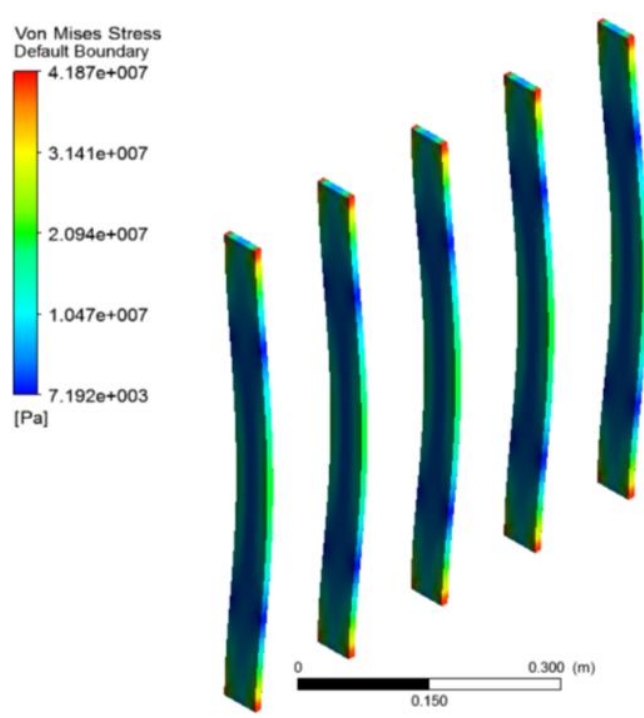

LWNSYYS

Figura 10 - Tensões de Von Mises nas barras verticais Fonte - Próprio autor.

Os mínimos valores de tensão encontrados estão localizados na região onde o momento fletor é zero. Ou seja, a uma distância do engastamento de 0,2113 vezes o comprimento da viga, que representa aproximadamente 137,35 mm dos apoios. A Figura 11 apresenta uma vista lateral da barra vertical a fim de identificar a região com as tensões mínimas. A tensão mínima neste modelo 0,007192 MPa, ou seja, praticamente zero.

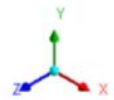

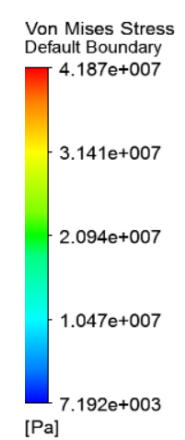

Os resultados das deformações na direção do eixo $\mathrm{x}$ obtidas na análise estão apresentados na Figura 12. Os resultados apresentados são referentes a um intervalo de tempo igual a 0,4 segundos. As máximas deformações em na direção $X$ estão localizadas na região central das barras, que coincide com a solução analítica do problema. As deformações mínimas estão localizadas na região dos apoios, pois se trata de engastamento perfeito. $O$ fator de escala foi exageradamente ajustado para uma melhor visualização das deformações nas barras. 


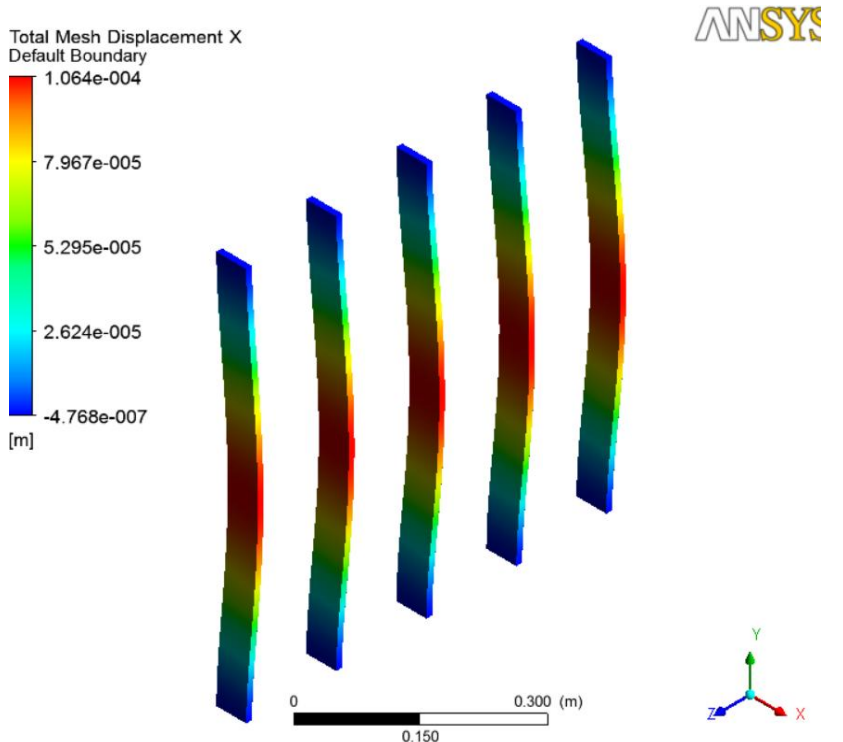

Figura 12 - Deformações na direção do eixo x nas barras verticais

Fonte - Próprio autor

Os resultados das deformações na direção do eixo z obtidas na análise estão apresentados na Figura 13. Os resultados apresentados são referentes à um intervalo de tempo igual a 0,4 segundos. A máxima deformação calculada na direção $Z$ foi de 0,01046 $\mathrm{mm}$, na região central da primeira barra lateral. As mínimas deformações na direção $Z$ estão localizadas nas extremidades das barras, ou seja, onde foi definido como condição de contorno de deslocamento igual a zero.

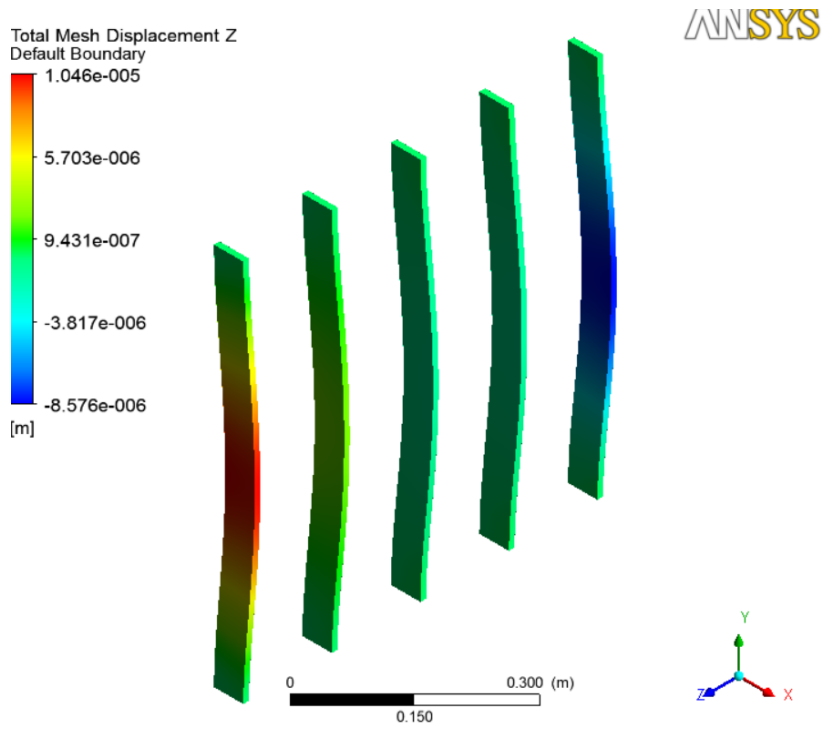

Figura 13 - Deformações na direção do eixo z nas barras verticais

Fonte - Próprio autor

\subsection{Resultados da ANÁlise de DinÂMICA dOS FLUIDOS}

Os resultados das velocidades estão apresentados na Figura 14. Foi definido um plano zx para visualização do perfil de velocidades. Essa Figura apresenta o perfil de velocidades resultantes da análise de dinâmica dos fluidos computacional.

A máxima velocidade calculada foi localizada na região próxima do canto vivo a montante da seção transversal das barras verticais. Essa velocidade possui a intensidade de $4,231 \mathrm{~m} \cdot \mathrm{s}^{-1}$. A região laranja da Figura identifica a formação dos vórtices. Esses vórtices são a principal fonte de vibração nas grades.

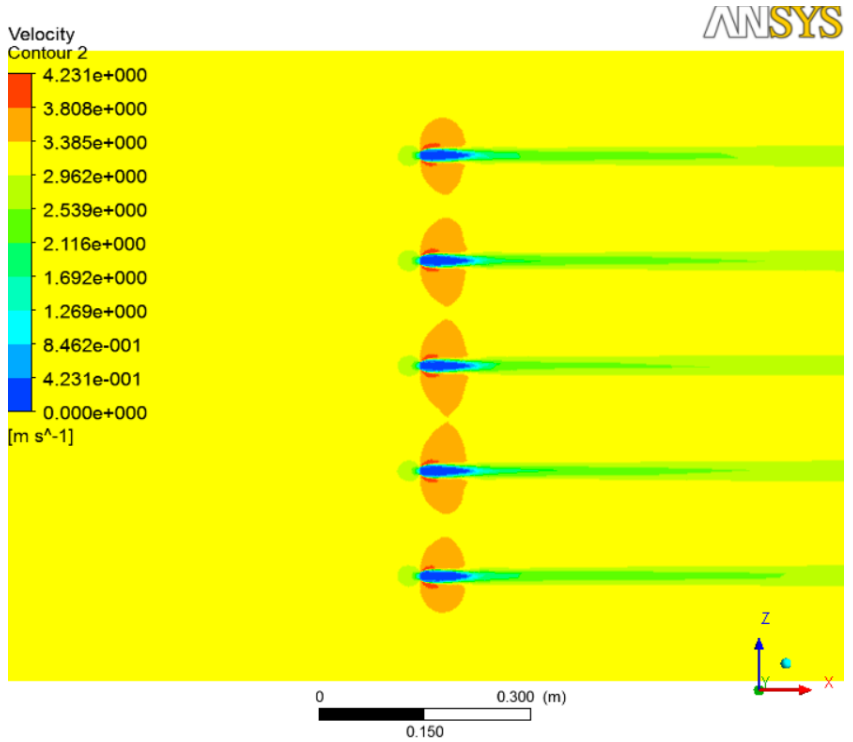

Figura 14 - Perfil de velocidades da análise de dinâmica dos fluidos (plano XZ localizado a $300 \mathrm{~mm}$ da origem - offset em y) Fonte - Próprio autor

A Figura 15 apresenta o detalhe dos vetores de velocidades resultantes da análise de dinâmica dos fluidos computacional. É possível notar que o vetor da máxima velocidade ocorre a um ângulo de aproximadamente 30 graus com a horizontal. A mínima velocidade calculada está localizada exatamente sobre a parede da estrutura da barra vertical. 


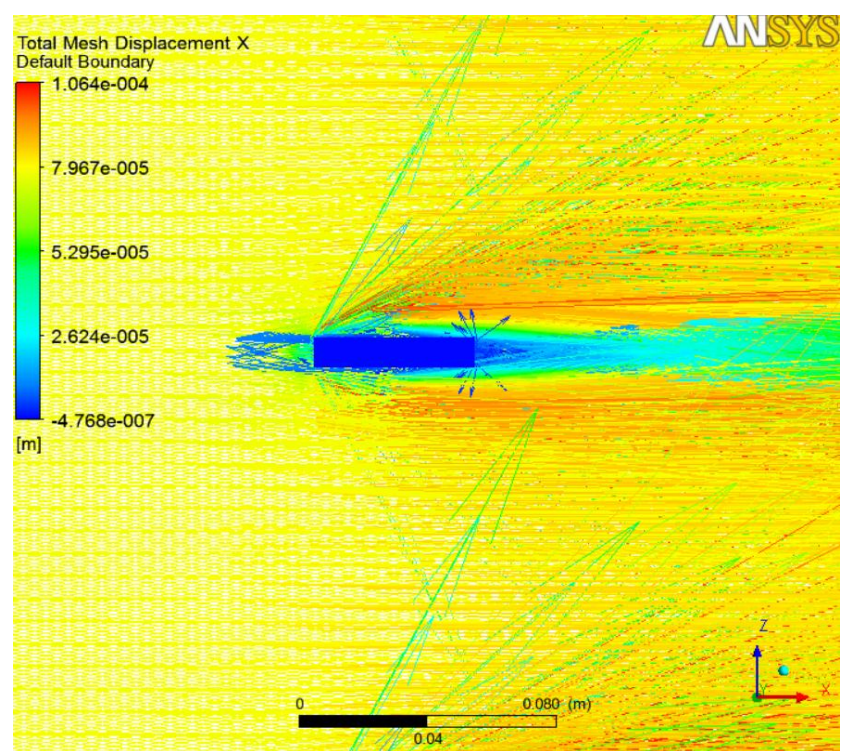

Figura 15 - Detalhe do perfil de velocidades da análise de dinâmica dos fluidos Fonte - Próprio autor

A Figura 16 mostra um zoom na região de interesse, ou seja, apresenta o detalhe das linhas de corrente quando o escoamento passa por uma barra vertical. No detalhe, é possível identificar a formação dos vórtices.

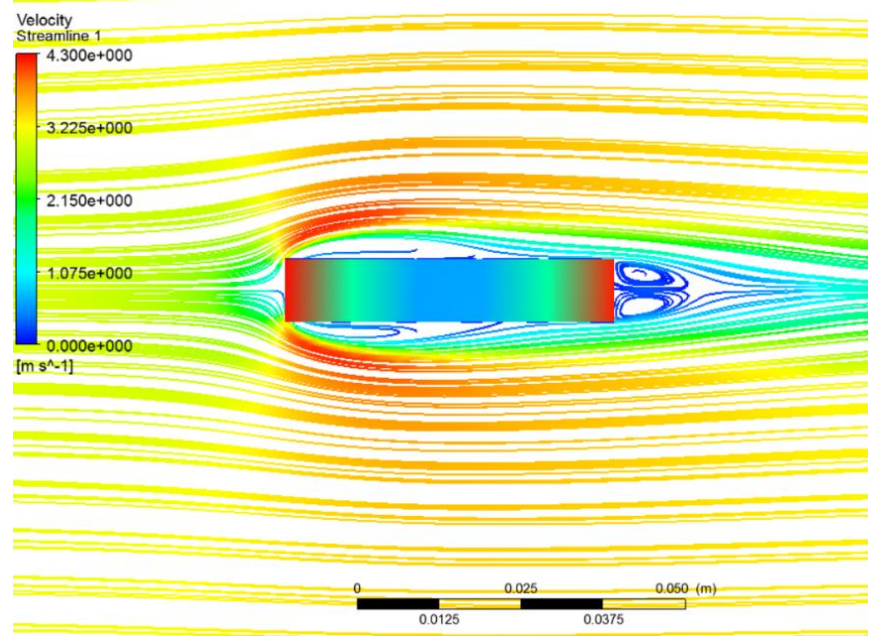

Figura 15 - Detalhe das linhas de corrente de velocidade da análise de dinâmica dos fluidos Fonte - Próprio autor

A Figura 16 apresenta o detalhe da distribuição de pressões na barra vertical devido ao escoamento. Tal Figura apresenta a máxima pressão que ocorre na face da barra em contato com a água. Segundo o cálculo o valor da máxima pressão é de $5.442 \mathrm{~Pa}$. A mínima pressão calculada foi de $-6.090 \mathrm{~Pa}$. A pressão mínima ocorre na região próxima aos cantos vivos a montante. Ou seja, devido ao descolamento da camada limite nesse ponto, formam-se vórtices que provocam uma região de sucção ou pressão negativa.

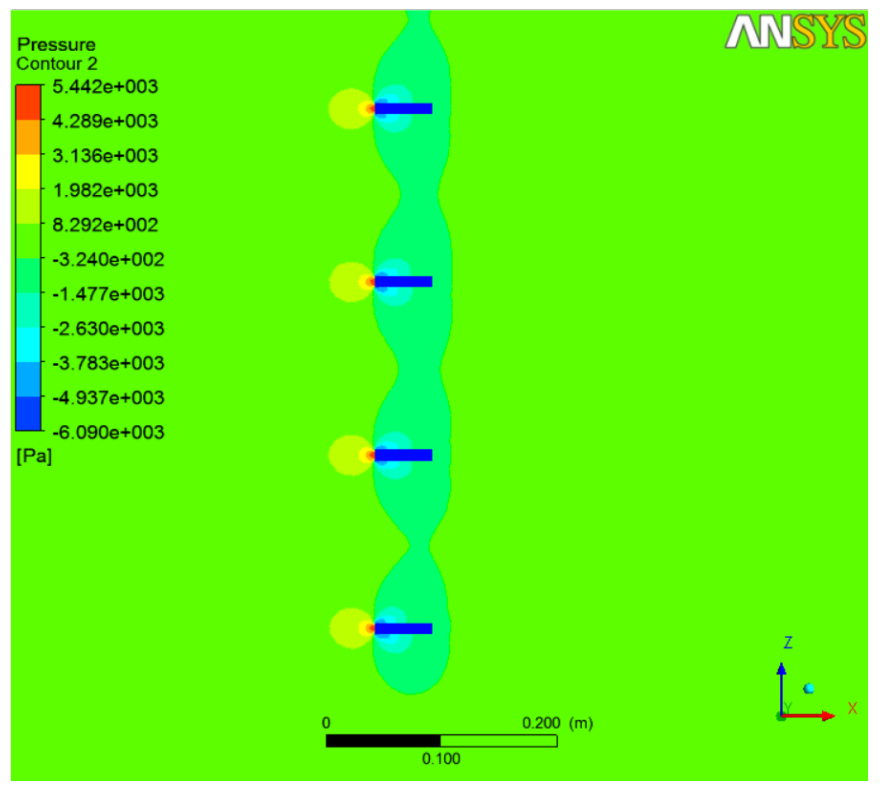

Figura 16 - Detalhe do perfil de pressões da análise de dinâmica dos fluidos Fonte - Próprio autor

\section{CONCLUSÕES}

Os resultados obtidos estão de acordo com o esperado, ou seja, os resultados de tensões se aproximaram com os cálculos analíticos.

O cálculo numérico apresentou uma tensão máxima de 41,86 MPa em t $=0,4 \mathrm{~s}$, na região engastada. Os resultados de tensão da análise numérica apresentaram uma variação de $6,51 \%$ a mais em relação à teórica devido ao fato de que a análise numérica considera, além da pressão devido à perda de carga de 3,0 m.c.a., a influência da pressão dinâmica exercida pelo escoamento. Além disso, a máxima tensão, ocorre em uma região de canto vivo.

Os resultados obtidos mostraram nitidamente a região de formação de vórtices do modelo. Esses vórtices são as principais fontes de vibração induzida na estrutura das barras. 
Assim, foi possível, através do software comercial ANSYS CFX 14, realizar-se uma simulação computacional com 0 objeto de avaliar qualitativamente a influência das velocidades dos fluidos de um escoamento de água interagindo com as barras verticais utilizando as técnicas de dinâmica de fluidos computacional (CFD), para um regime turbulento, modelo $k-\varepsilon$. O estudo mostrou que a simulação fluidodinâmica é uma importante ferramenta

\section{REFERÊNCIAS}

ABNT NBR 11213. Grade de Tomada d'Água para Instalação Hidráulica - Cálculo. Associação Brasileira de Normas Técnicas. 2001.

ANSYS CFX. User Manual, ANSYS-CFX 14. 2011.

BEHRING, A, G.; YEH, C. H. Flow-induced trashrack vibration. Harza Engineering Company, Chicago, Illinois, 1980.

BIRD, R.B.; STEWART, W.E.; LIGHTFOOT, E.N. Fenômenos de Transporte, LTC editora, 2a edição, Rio de Janeiro. 2004.

GHAMRY, H.; KATOPODIS, C. Numerical Investigation Of Turbulent Flow Through Bar Racks In Closed Conduits. 9th ISE 2012. Vienna.

HRIBERNIK, A.; FIKE, M.; HRIBERNIK, T. M. Economical Optimization of a Trashrack Design for a Hydropower Plant. University of Maribor, Faculty of Economics and Business Razlagova 14, Maribor, Slovenia. 2013.

HUANG, X.; VALERO, C.; EGUSQUIZA, E.; PRESAS, A.; GUARDO, A. Numerical and experimental analysis of the dynamic response of large submerged trash-racks. ELSEVIER. Computers \& Fluids 71 (2013) 54-64.

KERENYI, K. et al. Flow-Induced Multiple-Mode Vibrations of Lightly Damped Rectangular Trashrack Prisms. Institut für Konstruktiven Wasserbau, Technische Universität Wien, A-1040 Wien, Austria. 1997. a ser utilizada na elaboração de projetos mais complexos envolvendo tubulação cilíndrica, mais realísticas, característicos dos modelos com turbulência utilizados.

A utilização do software de elementos finitos ANSYS comprova a eficiência em obter resultados de análise de estruturas de forma precisa e rápida. Ou seja, garantir que as estruturas resistam as cargas aplicadas.
KOLKMAN, P. A.; JONGELING, T. H. G. Dynamic behaviour of hydraulic structures - Part A - Structures in flowing fluid. Delft Hydraulics. 2007.

NASCIMENTO, L. P.; SILVA, J. B. C.; GIUNTA, V. Model of Hydroelectric Power Plant Trashracks including Fluid-Structure Interaction, 17th International Congress of Mechanical Engineering, 2003. São Paulo-SP.

NGUYEN, T. D.; NAUDASCHER, E., FELLOW. Vibration of beams and Trash Racks in Parallel and Inclined Flows. J. Hydraul. Eng., 10.1061/(ASCE)0733-9429(1991)117:8(1056), 10561076. 1991.

PATANKAR, S.V. Numerical Heat Transfer and Fluid Flow. New York: Hemisphere. 1980.

SADRNEJAD, S. A. Hydrodynamics Induced Vibration to Trash-racks. Department of Civil Eng., K. N. Toosi University of Tech., Teheran, Iran. 2002.

SCHEUMANN, T. M. A Failure Analysis Case Study of the Fluid-Structural Interactions on Trashracks. A thesis submitted in partial fulfillment of the requirements for the degree of Master of Science. University of Washington. 2012.

\section{TIMOSHENKO, S. Vibrations Problems in} Engineering. Part I, Second Edition, 1937.

VERSTEEG, H. K.; MALALASEKERA, W. An introduction to computational fluid dynamics: the finite volume method. England: Longman Scientific \& Technical. 1995. 